\title{
Vascular architecture mapping for early detection of glioblastoma recurrence
}

\author{
Andreas Stadlbauer, PhD, ${ }^{1,2}$ Ilker Eyüpoglu, MD, ${ }^{1}$ Michael Buchfelder, MD, ${ }^{1}$ Arnd Dörfler, MD, ${ }^{3}$ \\ Max Zimmermann, MSc, ${ }^{1}$ Gertraud Heinz, MD, ${ }^{2}$ and Stefan Oberndorfer, MD ${ }^{4}$
}

Departments of ${ }^{1}$ Neurosurgery and ${ }^{3}$ Neuroradiology, University of Erlangen-Nürnberg, Erlangen, Germany; and ${ }^{2}$ Institute of Medical Radiology and ${ }^{4}$ Department of Neurology, University Clinic of St. Pölten, St. Pölten, Austria

OBJECTIVE Treatment failure and inevitable tumor recurrence are the main reasons for the poor prognosis of glioblastoma (GB). Gross-total resection at repeat craniotomy for GB recurrence improves patient overall survival but requires early and reliable detection. It is known, however, that even advanced MRI approaches have limited diagnostic performance for distinguishing tumor progression from pseudoprogression. The novel MRI technique of vascular architectural mapping (VAM) provides deeper insight into tumor microvascularity and neovascularization. In this study the authors evaluated the usefulness of VAM for the monitoring of GB patients and quantitatively analyzed the features of neovascularization of early- and progressed-stage GB recurrence.

METHODS In total, a group of 115 GB patients who received overall 374 follow-up MRI examinations after standard treatment were retrospectively evaluated in this study. The clinical routine MRI (cMRI) protocol at 3 Tesla was extended with the authors' experimental VAM approach, requiring 2 minutes of extra time for data acquisition. Custom-made MAT$\mathrm{LAB}$ software was used for calculation of imaging biomarker maps of macrovascular perfusion from perfusion cMRI as well as of microvascular perfusion and architecture from VAM data. Additionally, cMRI data were analyzed by two boardcertified radiologists in consensus. Statistical procedures included receiver operating characteristic (ROC) analysis to determine diagnostic performances for GB recurrence detection.

RESULTS Overall, cMRI showed GB recurrence in 89 patients, and in 28 of these patients recurrence was detected earlier with VAM data, by 1 (20 patients) or 2 (8 patients) follow-up examinations, than with cMRI data. The mean time difference between recurrence detection with VAM and CMRI data was 147 days. During this time period the mean tumor volume increased significantly ( $p$ <0.001) from 9.7 to $26.8 \mathrm{~cm}^{3}$. Quantitative analysis of imaging biomarkers demonstrated microvascular but no macrovascular hyperperfusion in early GB recurrence. Therefore, ROC analysis revealed superior diagnostic performance for VAM compared with cMRI.

CONCLUSIONS This study demonstrated that the targeted assessment of microvascular features using the VAM technique provided valuable information about early neovascularization activity in recurrent GB that is complementary to perfusion cMRI and may be helpful for earlier and more precise monitoring of patients suffering from GB. This VAM approach is compatible with existing cMRI protocols. Prospective clinical trials are necessary to investigate the clinical usefulness and potential benefit of increased overall survival with the use of VAM in patients with recurrent GB.

https://thejns.org/doi/abs/10.3171/2019.9.FOCUS19613

KEYWORDS glioblastoma; recurrence; neovascularization; MRI; vascular architecture

$\mathrm{G}$ LIOBLASTOMA (GB) is the most malignant and most common primary brain tumor in adults. ${ }^{21}$ It is characterized by rapid proliferation and highly aggressive and invasive growth into surrounding brain tissue. ${ }^{24}$
Vascular proliferation is a pathological hallmark of GB, which is among the most vascularized of all solid tumors. ${ }^{36}$ However, formation of new tumor vessels, also known as neovascularization, is highly defective, resulting in a dis-

ABBREVIATIONS AUC = area under the ROC curve; $\mathrm{CA}=$ contrast agent; $\mathrm{CBV}=$ cerebral blood volume; $\mathrm{CE}=$ contrast enhanced; cMRI = clinical routine MRI; $\mathrm{cNAWM}=$ contralateral normal appearing white brain matter; DSC = dynamic susceptibility contrast; EPI = echo-planar imaging; FN = false negative; FP = false positive; GB = glioblastoma; GE = gradient echo; MTI = microvessel type indicator; MVD = microvessel density; OS = overall survival; RANO = Response Assessment in Neuro-Oncology; ROC = receiver operating characteristic; $\mathrm{ROI}=$ region of interest; $\mathrm{SE}=$ spin echo; $\mathrm{TN}=$ true negative; $\mathrm{TP}=$ true positive; $\mathrm{T} 1 \mathrm{~W}=\mathrm{T} 1 \mathrm{weighted}$; $\mathrm{VAM}=\mathrm{vascular}$ architectural mapping; $\mathrm{VHL}=$ vascular hysteresis loop; $\mathrm{VSI}=$ vessel size index; $\mu \mathrm{CBV}=$ microvascular CBV.

SUBMITTED July 26, 2019. ACCEPTED September 4, 2019.

INCLUDE WHEN CITING DOI: 10.3171/2019.9.FOCUS19613. 
organized vasculature with abnormal capillary bed topology $^{26}$ and increased blood-brain barrier permeability. ${ }^{12}$ Neovascularization in GB has high clinical relevance as it correlates with biological aggressiveness, degree of malignancy, clinical recurrence, and postoperative survival. ${ }^{8}$

Despite ongoing progress in the therapeutic management of GB, the prognosis remains poor, with an overall survival (OS) of 14-15 months after maximum radical and safe resection, concomitant radiochemotherapy, and adjuvant chemotherapy. ${ }^{35} \mathrm{~A}$ main reason for the poor prognosis is the fact that GB treatment failure and recurrence are as yet inevitable and typically occur 6-7 months after surgery. Gross-total resection at repeat craniotomy of GB recurrence is associated with significantly longer $\mathrm{OS}^{3,25}$ compared with no surgery or subtotal resection ${ }^{22}$ and should, therefore, be performed whenever possible in patients with recurrent GB. However, early and reliable detection of GB recurrence is required to enable the most radical repeat resection of the tumor.

The diagnostic management of patients with GB is essentially based on MRI techniques. However, even advanced MRI approaches, including measures of perfusion and blood volume as well as improved criteria, such as those provided by the Response Assessment in NeuroOncology (RANO) group, ${ }^{38}$ are limited to reliably distinguishing active tumor tissue and tumor progression from reactive tissue and pseudoprogression, respectively. This ambiguity in GB recurrence detection requires repeated follow-up examinations that may result in loss of valuable time and further tumor progression. PET, especially with amino acid tracers, was recommended as a helpful adjunct to MRI for postoperative assessment of GB. ${ }^{7}$ PET, however, has several drawbacks, such as limited availability, requirement of an additional examination, and high costs. This makes PET less applicable for serial follow-up examinations in GB monitoring.

A novel MRI technique, termed vascular architectural mapping (VAM), provides deeper insight into tissue microvascularity and tumor neovascularization. ${ }^{4,10,20,37}$ The physical basis for this MRI-based assessment of microvasculature is the difference in sensitivity of gradient-echo (GE) and spin-echo (SE) MRI to magnetic susceptibility. ${ }^{4,10,20}$ As a result, the GE signals, which are commonly used in clinical routine MRI (cMRI) with perfusion, are dominated by larger vessel diameters (starting from 20 $\mu \mathrm{m}$, i.e., larger arterioles and venules), ${ }^{4}$ with reduced sensitivity to the smaller microvascular range. ${ }^{28}$ In contrast, SE signals exhibit a peak sensitivity to the microvasculature at a vessel diameter of around $10 \mu \mathrm{m},{ }^{4}$ including capillaries and both small arterioles and venules. Importantly, neovascularization at an early stage is clearly dominated by very thin vascular structures, which are therefore hardly detectable with the conventional GE perfusion MRI techniques used in cMRI protocols.

The purpose of this study was to evaluate the usefulness of VAM and SE perfusion MRI for the monitoring of GB patients after standard therapy. We investigated the diagnostic performance of cMRI and VAM for detection of tumor recurrence and quantitatively analyzed the features of neovascularization of early- and progressed-stage GB recurrence.

\section{Methods \\ Patient Selection}

A consecutively and prospectively populated institutional database was searched for patients who received follow-up MRI examinations after treatment of a GB (WHO grade IV) according to standard of care, i.e., maximal safe resection and radiotherapy with concomitant and adjuvant chemotherapy with temozolomide, ${ }^{34}$ between July 2015 and June 2019. Further inclusion criteria were 1) age $>18$ years and 2) available data obtained by use of our study MRI protocol from two or more follow-up examinations, if the cMRI data of the first follow-up revealed no recurrence of the tumor. A total of 115 patients (49 women, 66 men; age $58.7 \pm 12.0$ years; range $29-81$ years) fulfilled these criteria, and the data from a total of 374 follow-up MRI examinations were retrospectively evaluated in this study. The institutional review boards of the University of Erlangen and the University Clinic of St. Pölten approved this retrospective study. All patients gave written informed consent in accordance with the ethical standards of the Helsinki Declaration of 1975 and its later amendments. Written consent was obtained from all enrolled patients.

\section{MRI Data Acquisition}

Follow-up MRI examinations were performed every 3-4 months or on an unscheduled basis in case of clinical signs of tumor recurrence. MRI data acquisition was carried out on a 3-Tesla whole-body scanner (Tim Trio, Siemens) equipped with the standard 12-channel head coil. The cMRI protocol included, among others, the following sequences: 1) a FLAIR sequence; 2) a single-shot diffusion-weighted echo-planar imaging (DW-EPI) sequence $\left(\mathrm{b}=0,1000\right.$, and $\left.\left.2000 \mathrm{sec} / \mathrm{mm}^{2}\right) ; 3\right)$ a dynamic susceptibility contrast (DSC) perfusion MRI using a GE-EPI sequence (TR, $1740 \mathrm{msec}$; TE, $22 \mathrm{msec}$ ); and 4) pre- and post-contrast-enhanced (CE) T1-weighted (T1W) MRI. All sequences were performed in the axial orientation.

Additionally, for the VAM approach, a DSC perfusion MRI using an SE-EPI sequence (TR, $1740 \mathrm{msec}$; TE, 33 $\mathrm{msec}$ ) was performed in combination with a separate contrast agent (CA) injection. ${ }^{17,32}$ Both DSC perfusion examinations were performed with 60 dynamic measurements and administration of $0.1 \mathrm{mmol} / \mathrm{kg}$ body weight gadoterate meglumine (Dotarem, Guerbet) as the CA at a rate of $4 \mathrm{~mL} / \mathrm{sec}$ using an MR-compatible injector (Spectris, Medrad). The first DSC-MRI was obtained by using SE-EPIDSC perfusion MRI because unwanted CA leakage occurs to a lesser extent with this technique. ${ }^{32}$ Our strategies to minimize the probability of patient motions and differences in the time to first-pass peak, which may significantly affect the data evaluation, were described previously. ${ }^{32}$ Geometrical parameters were chosen that were identical for the 2 DSC perfusion sequences: in-plane resolution of $1.8 \times 1.8 \mathrm{~mm}$, slice thickness of $4 \mathrm{~mm}, 29$ slices, and generalized autocalibrating partially parallel acquisition (GRAPPA) factor of 2. The additional acquisition time for the VAM approach, i.e., the SE-DSC perfusion sequence, was 2 minutes. 


\section{MRI Data Analysis}

Analysis of cMRI data included calculation of maps of apparent diffusion coefficients from diffusion-weighted imaging (DWI) data as well as maps of cerebral blood volume (CBV) from the GE-DSC perfusion cMRI data via automatic identification of arterial input functions. ${ }^{2,29}$ Routine MRI data including these DWI and CBV maps were analyzed by at least two board-certified radiologists in consensus based on the updated RANO criteria for detection of recurrence of GB. ${ }^{38}$

Analysis of VAM data was performed using custommade MATLAB (MathWorks) software. VAM data postprocessing consisted of 4 steps: 1) calculation of maps of microvascular CBV $(\mu \mathrm{CBV})$ from the SE-DSC perfusion MRI data; 2) calculation of the so-called vascular hysteresis loop (VHL), ${ }^{32,33}$ i.e., the $\Delta \mathrm{R}_{2, \mathrm{GE}}$ versus $\left(\Delta \mathrm{R}_{2, \mathrm{SE}}\right)^{3 / 2}$ diagram, ${ }^{20,39}$ via fitting of the first CA bolus curves of both the GE- and SE-DSC perfusion data, respectively, using a previously described gamma-variate function ${ }^{11}$ (correction for remaining CA extravasation was performed as described previously); ${ }^{5,6,32} 3$ ) calculation of microvessel density (MVD) and vessel size index (VSI; i.e., the microvessel radius); ${ }^{18}$ and 4) calculation of the microvessel type indicator (MTI), which was previously ${ }^{32}$ defined as the area of the VHL with the sign of its rotational direction (i.e., a clockwise VHL direction was identified with a plus sign, and a counterclockwise with a minus sign). Based on previous studies, ${ }^{14,32}$ a positive MTI value (i.e., clockwise VHL) is associated with a vascular system that is dominated by arterioles, whereas a negative MTI value (i.e., counterclockwise VHL) is associated with venule- and capillary-like vessel components. For guidance of interpretation in the MTI maps, positive MTI values were assigned to warm colors and negative MTI values to cool colors. Consequently, maps of MTI enabled differentiation between supplying arterial (areas with warm colors) and draining capillary-venous (areas with cool colors) microvasculature. Furthermore, the more negative the MTI value, the stronger is the neovascular activity in the tumor. ${ }^{31}$ The VAM postprocessing was described in detail previously. ${ }^{32}$

\section{Statistics}

All data were expressed as mean $\pm \mathrm{SD}$. Areas of contrast enhancement on CE T1W images suspected as tumor recurrence were selected using manually defined regions of interest (ROIs). Additional ROIs were positioned in contralateral normal appearing white brain matter (cNAWM) and used as an internal reference. Imaging biomarker values for CBV, $\mu \mathrm{CBV}, \mathrm{MVD}$, VSI, and MTI were calculated for the ROIs. Based on evaluation of the imaging features seen on the cMRI and VAM data, respectively, obtained from all follow-up examinations during the whole study period, patient subgroups were retrospectively defined according to true-positive (TP), false-positive (FP), truenegative $(\mathrm{TN})$, or false-negative $(\mathrm{FN})$ results for detection of GB recurrence. The parameters for diagnostic performance (sensitivity, specificity, and accuracy) were calculated from these data. Additionally, the total tumor volumes were determined by CE T1W MRI for patients with recurrent GB.
Dedicated software (SPSS, IBM Corp.) was used for statistical evaluation. Differences in imaging biomarkers between subgroups of patients were determined using the general linear model method. A Dunnett T3 test was used as a post hoc procedure to be consistent with the assumption that homogeneity of variance was not met and for correction for multiple comparisons. Homogeneity of variance was tested using Levene's test. Intraindividual differences in imaging biomarker values between lesions and cNAWM as well as in tumor volume between follow-up examinations were compared using a Wilcoxon signed-rank test. Significance of differences in tumor volume between patient subgroups was calculated using a Mann-Whitney U-test, and $p$ values less than 0.05 were considered to indicate significance. Receiver operating characteristic (ROC) analysis was performed to calculate the area under the ROC curve (AUC) to determine the diagnostic performance of each imaging biomarker for GB recurrence detection.

\section{Results}

Of the 115 patients included in this study, 89 patients showed GB recurrence and 26 patients showed no recurrence during the study period. Treatment of the recurrent GB was initiated after detection in cMRI in accordance with clinical signs for recurrence and included a repeat craniotomy in 33 patients (37.1\%), second-line monotherapy with the antiangiogenic drug bevacizumab in 26 patients $(29.2 \%)$, a temozolomide rechallenge in 17 patients (19.1\%), repeat radiation therapy or repeat combined radiochemotherapy in 10 patients (11.2\%), and palliative care without further treatment of the tumor in 3 patients (3.4\%).

\section{Early Recurrence Detection in VAM}

Recurrence of the GB was detected simultaneously by both cMRI and VAM data from the same examination in 61 patients (68.5\% of all patients with recurrence). This subgroup of patients was termed "simultaneously TP." An illustrative case for this subgroup is depicted in Fig. 1A. In the remaining 28 patients with recurrence of the GB during the study period, recurrence was detected earlier in VAM data of follow-up examinations whose cMRI data showed no evidence for recurrence, i.e., cMRI was FN. Therefore, this subgroup was termed "early TP in VAM \& FN in cMRI." In these patients, recurrence was detected in the cMRI data of the next follow-up (in 20 patients; illustrative case in Fig. 2) or the follow-up after (8 patients, Fig. 3). The time differences in detail were $147 \pm 80$ days (range 34-286 days). During this time period the increase in tumor volume on CE T1W MRI was statistically significant $(\mathrm{p}<0.001)$, from $9.7 \pm 11.1 \mathrm{~cm}^{3}\left(0.2-37.0 \mathrm{~cm}^{3}\right)$ to $26.8 \pm 22.4 \mathrm{~cm}^{3}\left(0.6-84.7 \mathrm{~cm}^{3}\right)$. The degree of tumor volume increase ranged between $\mathrm{a}+13 \%$ and a 155 -fold increase and showed a 10-fold increase on average. The tumor volumes at the early follow-ups, at which only VAM revealed evidence for GB recurrence, were additionally significantly smaller $(p=0.004)$ than the tumor volumes of the simultaneously TP subgroup $(20.9 \pm 21.0$ $\mathrm{cm}^{3}$ [0.9-84.2 $\left.\mathrm{cm}^{3}\right]$; Fig. 4A). No further FN results were found for cMRI, and no FN cases were found for VAM. 

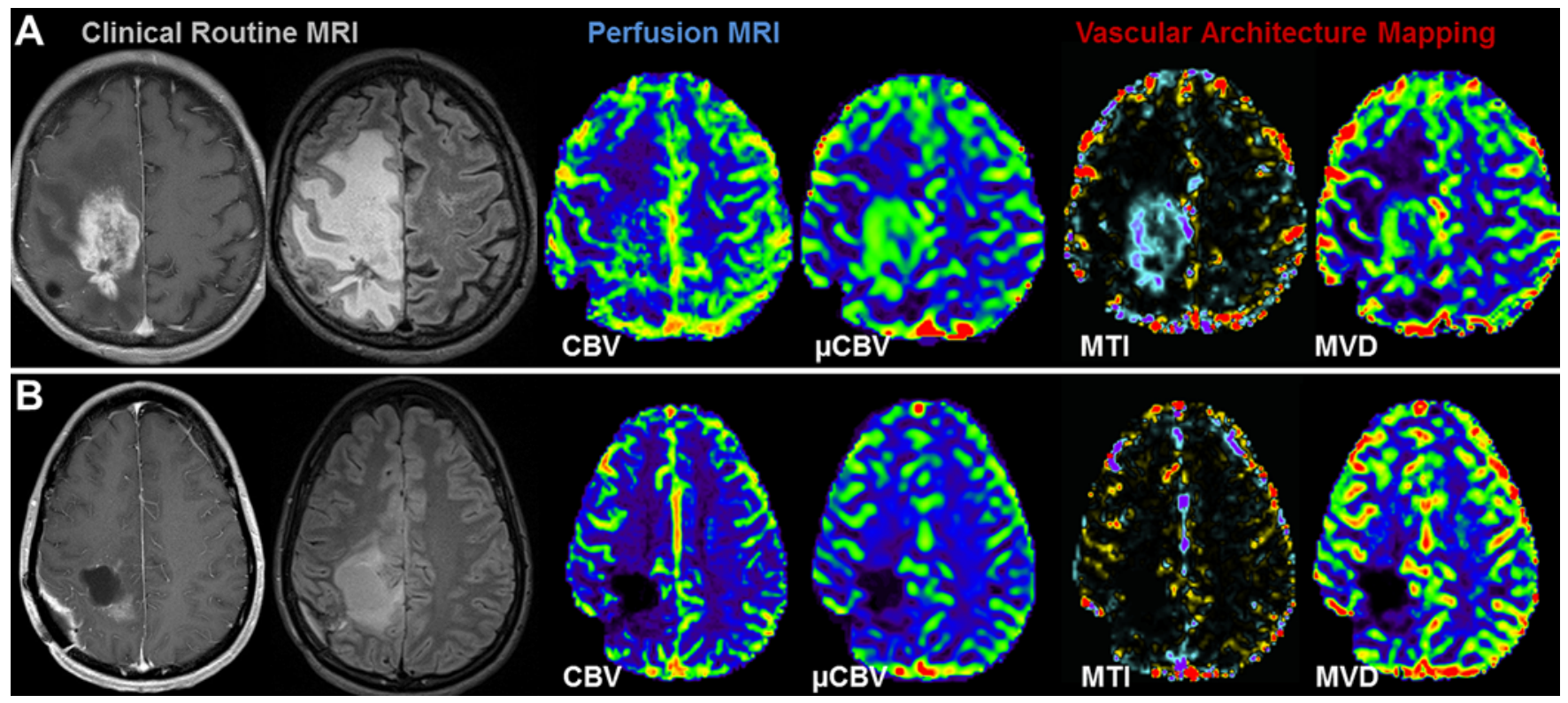

FIG. 1. A: Simultaneous TP detection of GB recurrence in both cMRI and VAM. cMRI, including anatomical sequences (CE T1W and FLAIR MRI) and macrovascular perfusion (CBV), and VAM biomarker maps of microvascular perfusion ( $\mu$ CBV), MTI, and MVD in a 54-year-old male patient clearly demonstrate recurrence of a GB. This patient received bevacizumab as second-line monotherapy. B: TN follow-up examination in both cMRI and VAM. The cMRI and VAM data of a 37-year-old female patient revealed pseudoprogression and no evidence for GB recurrence. This patient additionally showed no evidence for recurrence in the next 9 follow-up examinations carried out during the following 3 years.
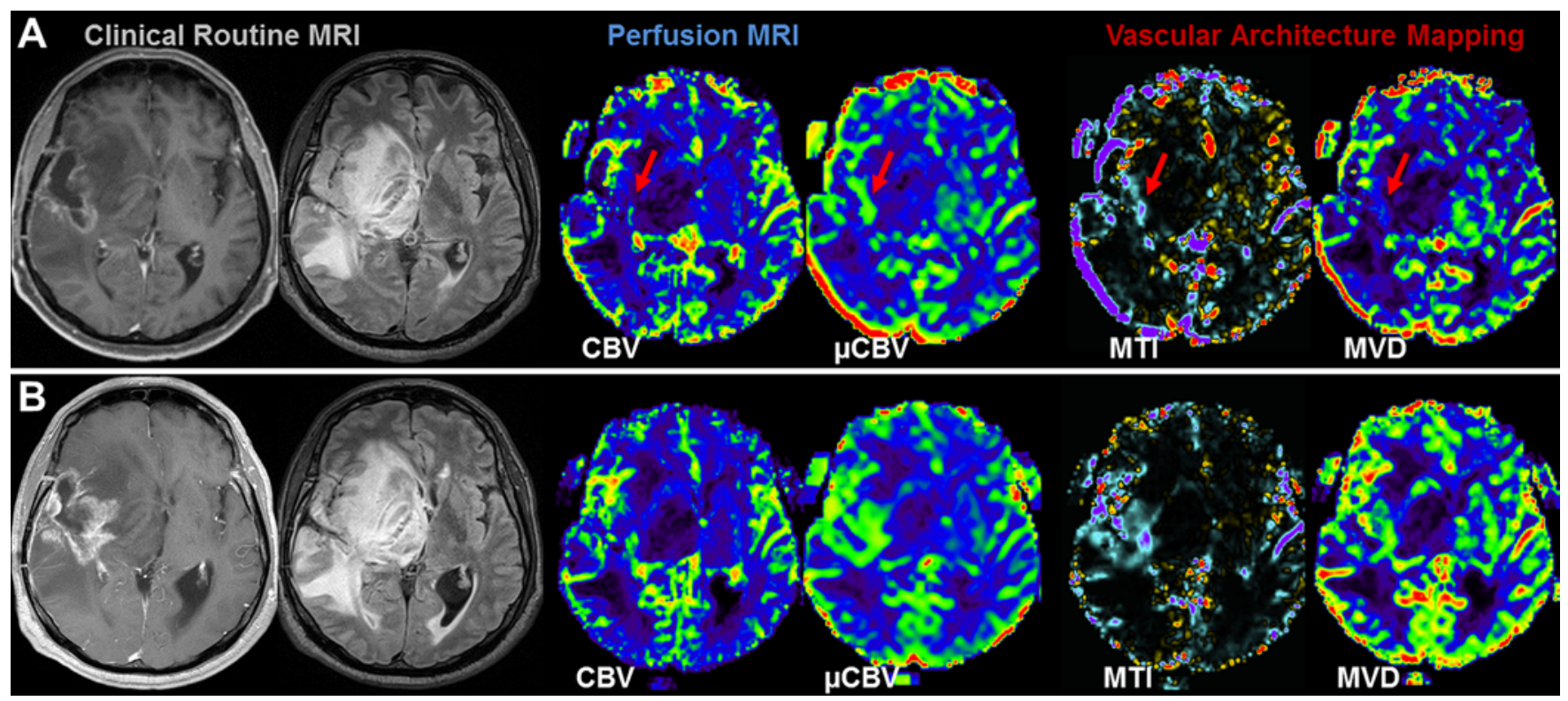

FIG. 2. Early TP detection of GB recurrence in VAM data and FN result in cMRI. A: cMRI, including anatomical sequences (CE T1W and FLAIR MRI) and macrovascular perfusion (CBV), and VAM biomarker maps of microvascular perfusion ( $\mu C B V), M T I$, and MVD of a 63-year-old male patient who was diagnosed with pseudoprogression in cMRI (no macrovascular hyperperfusion in $\mathrm{CBV}$, red arrow), but showed evidence for recurrence in VAM data (microvascular hyperperfusion in $\mu \mathrm{CBV}$ and indication of neovascularization in MTI, but not in MVD, red arrows) at the initial follow-up examination. B: At the subsequent follow-up examination 142 days later the patient showed clear signs of recurrence in CMRI data, and progression of the recurrent GB in VAM data. The tumor volume had increased by $89 \%$, from 15.6 to $29.4 \mathrm{~cm}^{3}$, within the 142 days. The patient received bevacizumab as second-line monotherapy. 

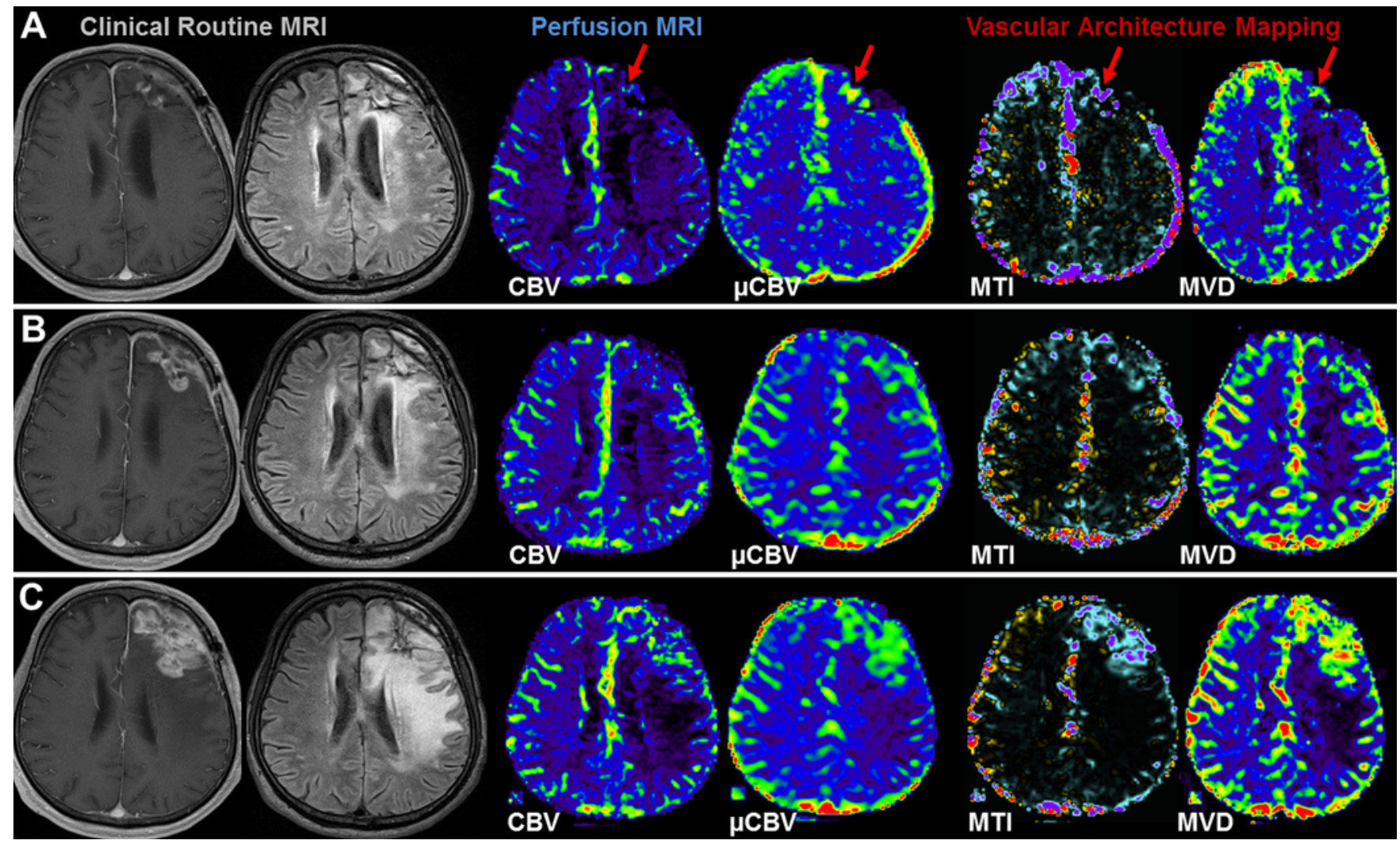

FIG. 3. Early TP detection of GB recurrence in VAM data and FN result in CMRI. A and B: CMRI, including anatomical sequences (CE T1W and FLAIR MRI) and macrovascular perfusion (CBV), and VAM biomarker maps of microvascular perfusion ( $\mu C B V)$, $\mathrm{MTI}$, and MVD of a 69-year-old male patient who was diagnosed with pseudoprogression in CMRI (no macrovascular hyperperfusion in CBV, red arrow) but showed evidence for recurrence in VAM data (microvascular hyperperfusion in $\mu$ CBV and indication of neovascularization in MTI and MVD, red arrows) at both the initial follow-up examination (A) and the subsequent follow-up examination 122 days later $(B)$. At the third follow-up examination another 95 days later $(\mathbf{C})$, the patient showed clear signs of recurrence in cMRI data and progression of the recurrent GB in VAM data, respectively. The tumor volume had increased 39-fold, from 17.3 to $67.8 \mathrm{~cm}^{3}$, within these in total 217 days. The patient underwent repeat craniotomy with subtotal resection and histopathological analysis of tissue specimens revealed GB.
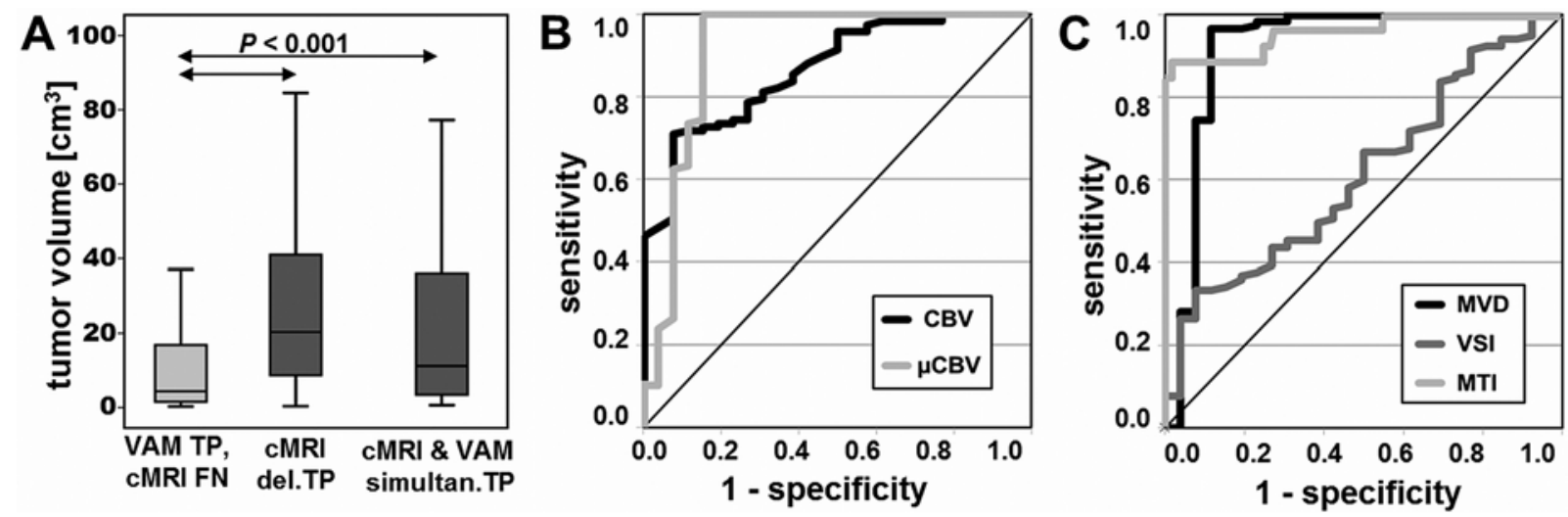

FIG. 4. A: Tumor volumes for the patient subgroups with an early TP result in VAM data and an FN finding in the cMRI data (VAM TP, cMRI FN, light gray box-and-whisker plot on the left) at the initial follow-up examination. The corresponding tumor volumes of the same patients at the subsequent follow-up with delayed TP result in cMRI (cMRI del. TP) are depicted in the dark gray box-and-whisker plot in the center. The dark gray box-and-whisker plot on the right depicts the tumor volumes for the patient subgroup with simultaneous (simultan.) TP findings in both CMRI and VAM data. ROC curves for differentiation of GB recurrence and pseudoprogression (B and C) illustrate diagnostic performance. B: Measures of macrovascular perfusion (CBV, black) and microvascular perfusion ( $\mu \mathrm{CBV}$, gray). C: Microvessel density (MVD, black), vessel size index (VSI, dark gray), and microvessel type indicator (MTI, light gray). MTI had the highest AUC (0.958) for detection of GB recurrence. 

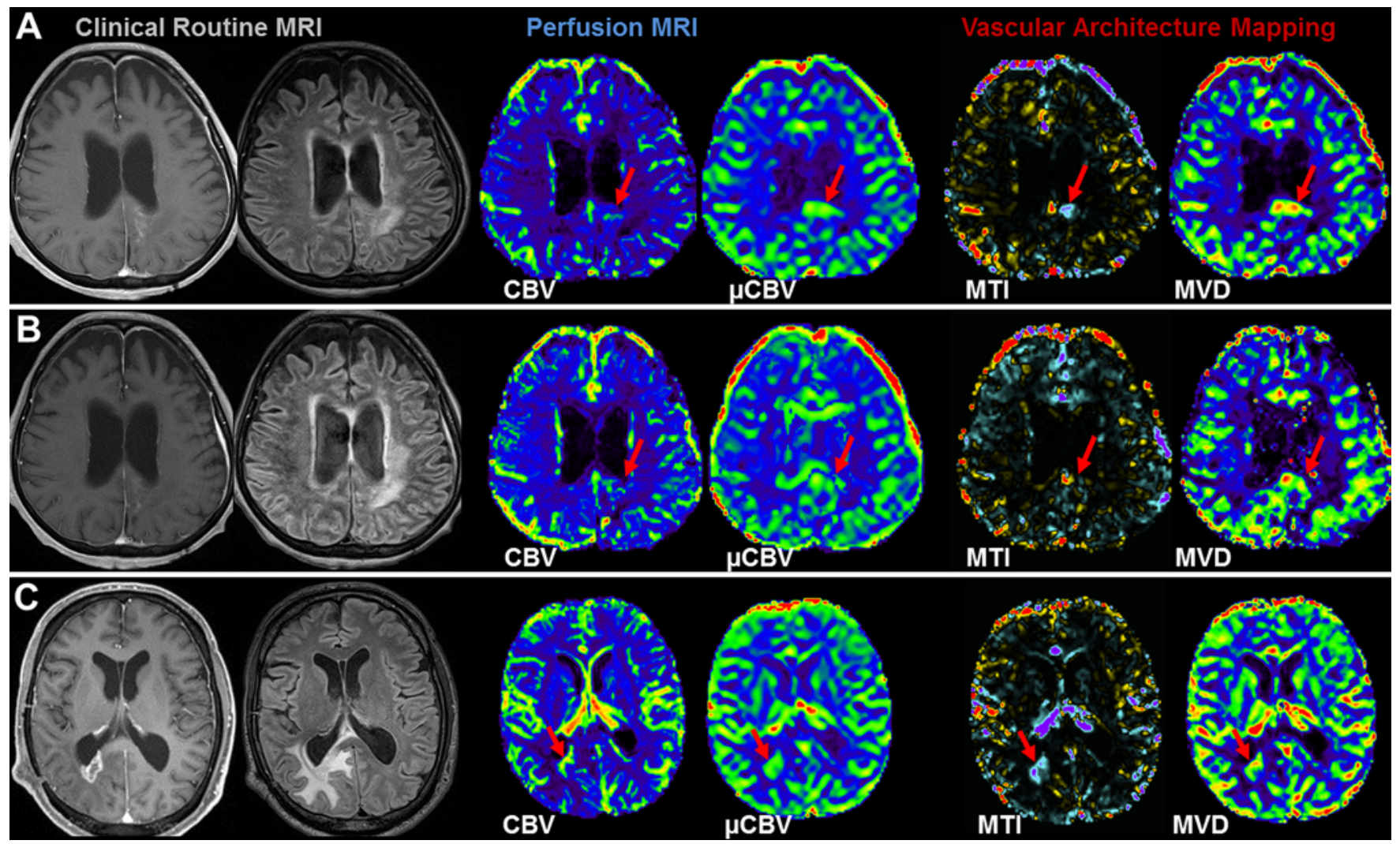

FIG. 5. An FP result for GB recurrence in VAM data and a TN finding in cMRI. A: CMRI, including anatomical sequences (CE T1W and FLAIR MRI) and macrovascular perfusion (CBV), and VAM biomarker maps of microvascular perfusion ( $\mu \mathrm{CBV}), \mathrm{MTI}$, and MVD of a 69-year-old female patient who was correctly diagnosed with pseudoprogression in CMRI (no macrovascular hyperperfusion in CBV). Signs for neovascularization in VAM data (red arrows) at this initial follow-up examination were falsely interpreted as recurrence, as it turned out at the subsequent follow-up examination 111 days later (B), at which the patient showed no signs of recurrence or progression in the CMRI and VAM data (red arrows). C: An FP result for GB recurrence in both cMRI and VAM data of a 52-year-old male patient. The patient received repeat craniotomy but histopathological analysis of tissue specimens revealed radiation necrosis and no vital tumor tissue (red arrows).

In the 26 patients who showed no recurrence during the study period, cMRI and VAM correctly revealed no recurrence (i.e., a TN result) for all follow-up examinations in 21 and 22 patients, respectively. An illustrative example for a TN result in both cMRI and VAM is depicted in Fig. 1B. However, FP results were found in 5 and 4 patients for cMRI and VAM, respectively. In all but 1 FP case, there correctly was no treatment of GB recurrence initiated, because clinical parameters showed no evidence for recurrence. The subsequent follow-up examinations of these patients ( 1 to 6 examinations over a time period of 4 months to 2 years) actually revealed no evidence for recurrence. Figure 5A and B illustrates imaging findings in a patient who showed clear signs for neovascularization in the initial follow-up in the VAM data but no obvious hyperperfusion in the cMRI data (Fig. 5A). The subsequent follow-up 4 months later (Fig. 5B) showed no signs for recurrence or progression and revealed that the initial findings were TN for cMRI and FN for VAM, respectively. The 1 FP case mentioned above, in which recurrence treatment was initiated, is depicted in Fig. 5C. In this patient, both cMRI and VAM data showed indications of recurrence. However, histological analysis of tissue specimens obtained during the repeat resection revealed radiation necrosis and no vital tumor tissue.

\section{Quantitative Evaluation of Imaging Biomarkers}

The values for the imaging biomarkers of cMRI (i.e., macrovascular CBV) and VAM (i.e., $\mu \mathrm{CBV}$, MVD, VSI, and MTI) for the patient subgroups are summarized in Table 1. Macrovascular CBVs in patients with an FN finding in cMRI were significantly lower $(\mathrm{p}<0.001)$ than those in both subgroups with TP findings in cMRI ("simultan TP" and "delayed TP" in Table 1), but were not significantly different from CBV values in TN and FP patients, as well as in cNAWM. These findings provided quantitative confirmation for absence of macrovascular hyperperfusion in early GB recurrence. CBV values for patients with FP findings in cMRI were significantly lower $(\mathrm{p}<0.05)$ than the values in both subgroups with TP findings in cMRI, and were not significantly different from the CBV values in cNAWM. As it turned out, this FP finding was mostly associated with small spots of high CBV from large vessels within the hyperintense region on the CE T1W MR images that were misinterpreted as GB recurrence.

The values of $\mu \mathrm{CBV}, \mathrm{MVD}$, and MTI in patients with 


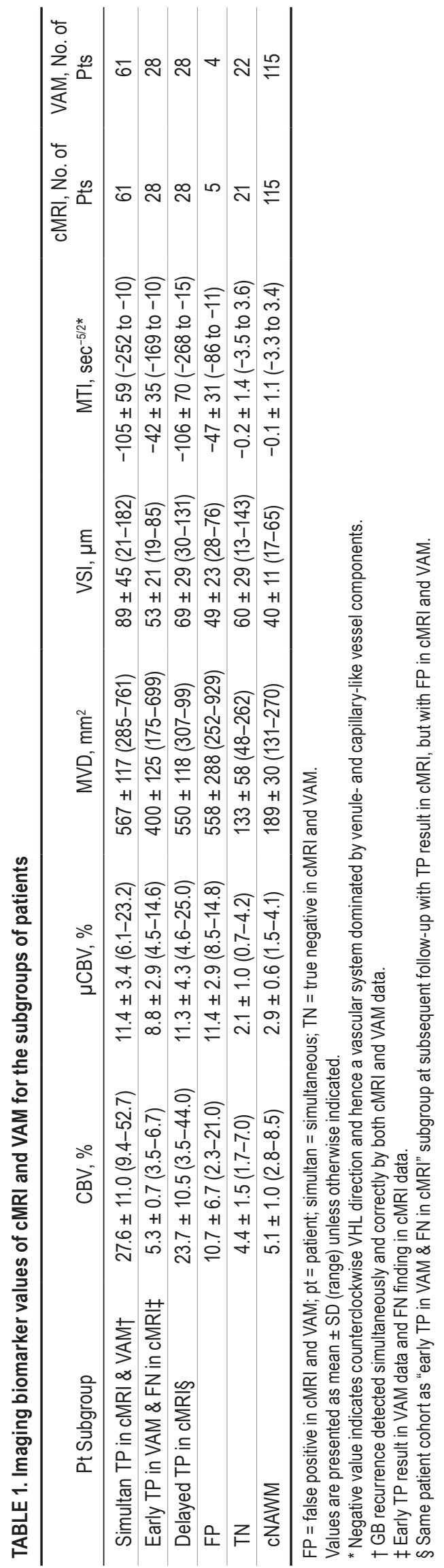

early TP findings in VAM were significantly lower $(\mathrm{p}<$ 0.05 for $\mu \mathrm{CBV} ; \mathrm{p} \leq 0.001$ for MVD and MTI) than the values in the other 2 subgroups with TP findings in VAM (Table 1) and were significantly higher than the values in the TN and FP cases as well as in cNAWM. These findings provide quantitative confirmation for microvascular hyperperfusion in early GB recurrence and a continued increase in neovascular activity during progression of the tumor. Biomarker values for FP cases in VAM, however, were not significantly different from values in the subgroups with TP findings in VAM and were significantly different from the values for cNAWM. This was a quantitative confirmation that there was indeed neovascular activity within the hyperintense region on the CE T1W MR images, but it remained unclear why no recurrent GBs have developed in the further disease course of these patients. Finally, we found that VSI was not useful for detection of GB recurrence, because we found no significant differences when comparing the subgroup values with each other or with the cNAWM values.

\section{Diagnostic Performance of Biomarkers for GB Recurrence Detection}

The findings of the quantitative analysis were confirmed by both the parameters for diagnostic performance and the ROC curve analysis results. Sensitivity, specificity, and accuracy for detection of GB recurrence were $0.685,0.808$, and 0.713 for cMRI, and 1.0, 0.846, and 0.965 for VAM, respectively. ROC curve analysis (Fig. 4B and C) revealed that MTI had the highest AUC $(0.958 ; \mathrm{p}<0.001)$ for detection of GB recurrence, followed by the VAM biomarkers MVD (AUC 0.919; $\mathrm{p}<0.001$ ) and $\mu$ CBV (AUC 0.912; $\mathrm{p}<$ 0.001 ), with very similar diagnostic performance. Macrovascular CBV showed slightly inferior diagnostic performance (AUC 0.866; $\mathrm{p}<0.001$ ), and VSI had the worst diagnostic performance for GB recurrence detection (AUC $0.614 ; \mathrm{p}=0.069$ ).

\section{Discussion}

In the present study, we extended our cMRI protocol for monitoring of GB patients with an approach for assessment of microvascular perfusion and architecture. Our main findings were threefold: 1) VAM allowed for early detection of GB recurrence, prior to cMRI; 2) early GB recurrence showed microvascular but no macrovascular hyperperfusion; and 3) VAM showed superior diagnostic performance compared with cMRI.

\section{Early GB Recurrence Detection With VAM}

We demonstrated that our VAM approach can provide additional information about tumor vasculature and neovascularization that is complementary to the information from routine perfusion MRI and may be helpful for early detection of GB recurrence. For tumors with very small volume $\left(1-2 \mathrm{~mm}^{3}\right)$, the supply of oxygen and nutrients from neighboring capillaries via vascular cooption ${ }^{16}$ is sufficient because the diffusion distance of oxygen is $100-200 \mu \mathrm{m} .{ }^{19}$ To grow beyond this size, generation of new blood vessels toward the tumor, i.e., neovascularization, is required. ${ }^{915}$ Mathivet et al. ${ }^{23}$ observed a progres- 
sive increase in vessel diameters during GB development in an orthotopic mouse model. VAM focuses precisely on these very thin vascular structures from early neovascularization and thereby closes the information gap in data from clinical routine perfusion MRI, which has very limited sensitivity to microvasculature. The reason for this is the high sensitivity of the SE-DSC perfusion MRI for microvessels with a diameter around $10 \mu \mathrm{m}$. This phenomenon has been well known in MR physics since the 1990s but was not applied in routine clinical use until now. Here, we demonstrated that the VAM approach is compatible with cMRI protocols and requires only 2 minutes of extra time for data acquisition.

\section{Microvascular but No Macrovascular Hyperperfusion in Early Recurrence}

Our quantitative analysis of cMRI and VAM imaging biomarkers demonstrated that early recurrence of GB is dominated by formation of small vascular structures, as already known and previously shown for untreated GB. ${ }^{23}$ $\mathrm{CBV}$ of the macrovasculature in early GB recurrence was found not to be significantly different from both pseudoprogression and cNAWM but was significantly lower than the CBV of progression-stage GB recurrence. On the other hand, $\mu \mathrm{CBV}, \mathrm{MVD}$, and MTI values for early-stage GB recurrence were significantly higher than the values for both pseudoprogression and cNAWM. Therefore, conventional GE-DSC perfusion MRI alone is not useful for detection of early-stage GB recurrence, and SE-DSC perfusion MRI should be considered for future modifications of the RANO criteria. ${ }^{38}$

\section{Superior Diagnostic Performance of VAM}

Repeat craniotomy is the first and best option for treating GB recurrence, but this treatment requires early and reliable recurrence detection. In this study, repeat craniotomy was possible in 33 of 89 patients (37.1\%) after (partly delayed) detection of GB recurrence in cMRI. For the remaining 56 patients with GB recurrence, only the second best alternatives for GB recurrence treatment (e.g., bevacizumab therapy) were available. The low percentage of repeat craniotomies demonstrates that the cMRI methods for GB patient monitoring were not sufficiently accurate. The accuracy of cMRI (0.713) was clearly lower than that of VAM (0.965). Detection of early-stage GB recurrence with VAM was associated with a significantly smaller tumor volume than detection with cMRI. However, it has to be established in future studies whether the detection of smaller-volume tumors is associated with a higher resection rate and an OS benefit.

The diagnostic performances of $\mu \mathrm{CBV}, \mathrm{MVD}$, and MTI were very similar in terms of AUC of ROC analyses. This opens the door for the application of our VAM approach in the clinical routine because $\mu \mathrm{CBV}$ can be easily calculated from SE-DSC perfusion MRI data and requires no sophisticated postprocessing software tools.

\section{Study Limitations}

A limitation of our VAM approach is the requirement for additional DSC-MRI perfusion sequences (SE-DSC) and a second $\mathrm{CA}$ injection. On the other hand, this ensures that the routine GE-DSC perfusion MRI sequence is kept unchanged regarding spatial and temporal resolution. However, our approach allows the acquisition of VAM data with high signal-to-noise ratio, high spatial resolution, and coverage of the whole brain, which is mandatory for MR perfusion examinations performed with cMRI. These features are especially important for detection of small or multicentric lesions. The commonly used combined simultaneous GE-SE-DSC perfusion sequence, $, 1,13,14,27,30$ however, does not meet these requirements because of insufficient spatial coverage (maximum of 12 sections) and resolution, which necessitates performing the routine perfusion separately and consequently requires a further CA injection. Additionally, the combined simultaneous GE-SE-DSC perfusion sequence was performed with a double dose or more of CA, $1,13,14,20,27,30$ which resulted in the application of up to a triple dose. Furthermore, this study was limited due to its retrospective design. Prospective clinical trials are necessary to investigate the clinical usefulness and potential OS benefit of VAM for patients with recurrent GB.

\section{Conclusions}

This study demonstrated that the targeted assessment of microvascular features using the VAM technique provided valuable information about early neovascularization activity in recurrent GB that is complementary to the information provided by perfusion imaging in cMRI and may be helpful for earlier and more precise monitoring of patients suffering from GB. Our VAM approach is compatible with existing MRI protocols for routine clinical diagnosis of GB recurrence, and an extension of this technique is straightforward if VAM data postprocessing remain limited to the calculation of $\mu \mathrm{CBV}$.

\section{Acknowledgments}

This work was supported by the German Research Foundation (Deutsche Forschungsgemeinschaft, DFG), grant numbers STA 1331/3-1 to Prof. Dr. Andreas Stadlbauer and DO 721/9-1 to Prof. Dr. Arnd Dörfler; and by the ELAN program (Erlanger Leistungsbezogene Anschubfinanzierung und Nachwuchsförderung) grant number 14-05-21-1-Stadlbauer to Prof. Dr. Andreas Stadlbauer.

\section{References}

1. Batchelor TT, Gerstner ER, Emblem KE, Duda DG, Kalpathy-Cramer J, Snuderl M, et al: Improved tumor oxygenation and survival in glioblastoma patients who show increased blood perfusion after cediranib and chemoradiation. Proc Natl Acad Sci U S A 110:19059-19064, 2013

2. Bjørnerud A, Emblem KE: A fully automated method for quantitative cerebral hemodynamic analysis using DSC-MRI. J Cereb Blood Flow Metab 30:1066-1078, 2010

3. Bloch O, Han SJ, Cha S, Sun MZ, Aghi MK, McDermott MW, et al: Impact of extent of resection for recurrent glioblastoma on overall survival: clinical article. J Neurosurg 117:1032-1038, 2012

4. Boxerman JL, Hamberg LM, Rosen BR, Weisskoff RM: MR contrast due to intravascular magnetic susceptibility perturbations. Magn Reson Med 34:555-566, 1995

5. Boxerman JL, Prah DE, Paulson ES, Machan JT, Bedekar D, Schmainda KM: The role of preload and leakage correction 
in gadolinium-based cerebral blood volume estimation determined by comparison with MION as a criterion standard. AJNR Am J Neuroradiol 33:1081-1087, 2012

6. Boxerman JL, Schmainda KM, Weisskoff RM: Relative cerebral blood volume maps corrected for contrast agent extravasation significantly correlate with glioma tumor grade, whereas uncorrected maps do not. AJNR Am J Neuroradiol 27:859-867, 2006

7. Buchmann N, Gempt J, Ryang YM, Pyka T, Kirschke JS, Meyer B, et al: Can early postoperative O-(2- ${ }^{18 F}$ fluoroethyl)L-tyrosine positron emission tomography after resection of glioblastoma predict the location of later tumor recurrence? World Neurosurg 121:e467-e474, 2019

8. Burger PC, Vogel FS, Green SB, Strike TA: Glioblastoma multiforme and anaplastic astrocytoma. Pathologic criteria and prognostic implications. Cancer 56:1106-1111, 1985

9. Carmeliet P, Jain RK: Angiogenesis in cancer and other diseases. Nature 407:249-257, 2000

10. Dennie J, Mandeville JB, Boxerman JL, Packard SD, Rosen BR, Weisskoff RM: NMR imaging of changes in vascular morphology due to tumor angiogenesis. Magn Reson Med 40:793-799, 1998

11. Ducreux D, Buvat I, Meder JF, Mikulis D, Crawley A, Fredy $\mathrm{D}$, et al: Perfusion-weighted MR imaging studies in brain hypervascular diseases: comparison of arterial input function extractions for perfusion measurement. AJNR Am J Neuroradiol 27:1059-1069, 2006

12. Dvorak HF: Rous-Whipple Award Lecture. How tumors make bad blood vessels and stroma. Am J Pathol 162:17471757,2003

13. Eichner C, Jafari-Khouzani K, Cauley S, Bhat H, Polaskova $\mathrm{P}$, Andronesi OC, et al: Slice accelerated gradient-echo spinecho dynamic susceptibility contrast imaging with blipped CAIPI for increased slice coverage. Magn Reson Med 72:770-778, 2014

14. Emblem KE, Mouridsen K, Bjornerud A, Farrar CT, Jennings D, Borra RJ, et al: Vessel architectural imaging identifies cancer patient responders to anti-angiogenic therapy. Nat Med 19:1178-1183, 2013

15. Folkman J, Kalluri R: Cancer without disease. Nature 427:787, 2004

16. Hardee ME, Zagzag D: Mechanisms of glioma-associated neovascularization. Am J Pathol 181:1126-1141, 2012

17. Hsu YY, Yang WS, Lim KE, Liu HL: Vessel size imaging using dual contrast agent injections. J Magn Reson Imaging 30:1078-1084, 2009

18. Jensen JH, Lu H, Inglese M: Microvessel density estimation in the human brain by means of dynamic contrast-enhanced echo-planar imaging. Magn Reson Med 56:1145-1150, 2006

19. Katayama Y, Uchino J, Chihara Y, Tamiya N, Kaneko Y, Yamada T, et al: Tumor neovascularization and developments in therapeutics. Cancers (Basel) 11:316, 2019

20. Kiselev VG, Strecker R, Ziyeh S, Speck O, Hennig J: Vessel size imaging in humans. Magn Reson Med 53:553-563, 2005

21. Louis DN, Perry A, Reifenberger G, von Deimling A, Figarella-Branger D, Cavenee WK, et al: The 2016 World Health Organization Classification of Tumors of the Central Nervous System: a summary. Acta Neuropathol 131:803-820, 2016

22. Lu VM, Jue TR, McDonald KL, Rovin RA: The survival effect of repeat surgery at glioblastoma recurrence and its trend: a systematic review and meta-analysis. World Neurosurg 115:453-459.e3, 2018

23. Mathivet T, Bouleti C, Van Woensel M, Stanchi F, Verschuere T, Phng LK, et al: Dynamic stroma reorganization drives blood vessel dysmorphia during glioma growth. EMBO Mol Med 9:1629-1645, 2017

24. Onishi M, Ichikawa T, Kurozumi K, Date I: Angiogenesis and invasion in glioma. Brain Tumor Pathol 28:13-24, 2011

25. Perrini P, Gambacciani C, Weiss A, Pasqualetti F, Delishaj D,
Paiar F, et al: Survival outcomes following repeat surgery for recurrent glioblastoma: a single-center retrospective analysis. J Neurooncol 131:585-591, 2017

26. Pries AR, Höpfner M, le Noble F, Dewhirst MW, Secomb TW: The shunt problem: control of functional shunting in normal and tumour vasculature. Nat Rev Cancer 10:587593,2010

27. Schmainda KM, Rand SD, Joseph AM, Lund R, Ward BD, Pathak AP, et al: Characterization of a first-pass gradientecho spin-echo method to predict brain tumor grade and angiogenesis. AJNR Am J Neuroradiol 25:1524-1532, 2004 (Erratum in AJNR Am J Neuroradiol 26:686, 2005)

28. Schmiedeskamp H, Straka M, Newbould RD, Zaharchuk G, Andre JB, Olivot JM, et al: Combined spin- and gradientecho perfusion-weighted imaging. Magn Reson Med 68:3040, 2012

29. Smith AM, Grandin CB, Duprez T, Mataigne F, Cosnard G: Whole brain quantitative CBF, CBV, and MTT measurements using MRI bolus tracking: implementation and application to data acquired from hyperacute stroke patients. J Magn Reson Imaging 12:400-410, 2000

30. Sorensen AG, Batchelor TT, Zhang WT, Chen PJ, Yeo P, Wang M, et al: A "vascular normalization index" as potential mechanistic biomarker to predict survival after a single dose of cediranib in recurrent glioblastoma patients. Cancer Res 69:5296-5300, 2009

31. Stadlbauer A, Gruber S, Nimsky C, Fahlbusch R, Hammen $\mathrm{T}$, Buslei R, et al: Preoperative grading of gliomas by using metabolite quantification with high-spatial-resolution proton MR spectroscopic imaging. Radiology 238:958-969, 2006

32. Stadlbauer A, Zimmermann M, Heinz G, Oberndorfer S, Doerfler A, Buchfelder M, et al: Magnetic resonance imaging biomarkers for clinical routine assessment of microvascular architecture in glioma. J Cereb Blood Flow Metab 37:632643, 2017

33. Stadlbauer A, Zimmermann M, Oberndorfer S, Doerfler A, Buchfelder M, Heinz G, et al: Vascular hysteresis loops and vascular architecture mapping in patients with glioblastoma treated with antiangiogenic therapy. Sci Rep 7:8508, 2017

34. Stupp R, Hegi ME, Mason WP, van den Bent MJ, Taphoorn MJ, Janzer RC, et al: Effects of radiotherapy with concomitant and adjuvant temozolomide versus radiotherapy alone on survival in glioblastoma in a randomised phase III study: 5-year analysis of the EORTC-NCIC trial. Lancet Oncol 10:459-466, 2009

35. Stupp R, Mason WP, van den Bent MJ, Weller M, Fisher B, Taphoorn MJB, et al: Radiotherapy plus concomitant and adjuvant temozolomide for glioblastoma. N Engl J Med 352:987-996, 2005

36. Takano S, Yamashita T, Ohneda O: Molecular therapeutic targets for glioma angiogenesis. J Oncol 2010:351908, 2010

37. Weisskoff RM, Zuo CS, Boxerman JL, Rosen BR: Microscopic susceptibility variation and transverse relaxation: theory and experiment. Magn Reson Med 31:601-610, 1994

38. Wen PY, Macdonald DR, Reardon DA, Cloughesy TF, Sorensen AG, Galanis E, et al: Updated response assessment criteria for high-grade gliomas: Response Assessment in Neuro-Oncology Working Group. J Clin Oncol 28:19631972,2010

39. Xu C, Kiselev VG, Möller HE, Fiebach JB: Dynamic hysteresis between gradient echo and spin echo attenuations in dynamic susceptibility contrast imaging. Magn Reson Med 69:981-991, 2013

\section{Disclosures}

The authors report no conflict of interest concerning the materials or methods used in this study or the findings specified in this paper. 


\section{Author Contributions}

Conception and design: Stadlbauer, Oberndorfer. Acquisition of data: Stadlbauer, Dörfler, Heinz, Oberndorfer. Analysis and interpretation of data: Stadlbauer, Eyüpoglu, Zimmermann, Oberndorfer. Drafting the article: Stadlbauer. Critically revising the article: all authors. Reviewed submitted version of manuscript: all authors. Approved the final version of the manuscript on behalf of all authors: Stadlbauer. Statistical analysis: Stadlbauer, Zimmermann. Administrative/technical/material support: Stadlbauer, Oberndorfer. Study supervision: Stadlbauer, Buchfelder, Dörfler, Heinz, Oberndorfer.

\section{Correspondence}

Andreas Stadlbauer: University of Erlangen-Nürnberg, Erlangen, Germany. andi@nmr.at. 